\title{
THE CONJUGACY PROBLEM FOR THE GROUP OF ANY TAME ALTERNATING KNOT IS SOLVABLE ${ }^{1}$
}

\author{
K. I. APPEL AND P. E. SCHUPP
}

\begin{abstract}
The theorem of the title is proved by using the techniques of Weinbaum along with the prime decomposition of knots and an extension of some small cancellation techniques of Lyndon and Schupp.
\end{abstract}

The argument to prove the theorem of the title is based on the proof of Weinbaum [6] for prime alternating knots which in turn employs the methods of Lyndon [2] and Schupp [5]. To save repetition, familiarity with Weinbaum's paper is assumed. Knot, in this paper, will always mean tame knot in general position with respect to a projection plane (i.e. all multiple points of the projection are isolated double points of which there are finitely many).

If $K$ is a knot then $K$ has a projection due to Schubert [4] which may be described as follows. Start with a circle, which we will call the Schubert circle, with some $k$ disjoint small open arcs deleted. Around the exterior of the circle place disjoint projections of $k$ prime knots each with a small open arc (not including a double point) deleted. Join the endpoints of the arcs on the prime projections pairwise to the endpoints of the open arcs on the circle without introducing further double points.

We also want to deal with knot projections having parts arranged around a fixed circle. We need a condition weaker than primeness on the parts.

Received by the editors August 11, 1971.

AMS 1970 subject classifications. Primary 20F10; Secondary 20F05, 20E40, 55A05, $55 \mathrm{~A} 25$.

Key words and phrases. Conjugacy problem, word problem, fundamental group, knot group, knot diagram, tame knot, prime knot, alternating knot, generators, relations.

${ }^{1}$ This proof is simpler than a proof announced by Appel [1] which was based on a Wirtinger presentation, and therefore that proof will not be published. The authors are indebted to Professor Wolfgang Haken for many helpful suggestions.

This research was supported by National Science Foundation Grant GP 23707. 
Definition. A knot projection is called elementary if (in Weinbaum's terminology):

(1) any vertex is on the boundary of four distinct domains;

(2) any two domains have at most one edge in common.

(Our definition of "elementary" is exactly the same as Weinbaum's definition of "common".)

Being elementary is a property of projections while being prime is a property of knots independent of particular projections.

If $K$ is a prime knot, then any projection of $K$ having a minimal number of crossings is elementary. Composite knots may have elementary projections. (We conjecture that if a projection is both alternating and elementary then it is a projection of a prime knot.)

We define below a "standard projection" of a knot. The standard projection will consist of elementary parts arranged around a fixed circle. Based on Schubert's ideas, we give an algorithm which, starting from any projection of a knot $K$, produces a standard projection of $K$. For the purposes of the present paper there are two advantages of replacing prime by elementary. First, given any alternating projection, the standard projection produced is also alternating. Second, the given procedure is clearly effective.

Definition. Let $\pi$ be a projection of a knot $K$. Let $\Gamma$ be a simple closed curve which intersects $\pi$ in precisely two points and such that there are vertices of $\pi$ both interior to and exterior to $\Gamma$. Then $\Gamma$ is called a separating curve for $\pi$.

Definition. Let $\pi$ be a projection of a knot $K$. We call $\pi$ standard if

(i) any vertex of $\pi$ is on the boundary of four distinct domains;

(ii) there exists a bounded domain $X_{1}$ of $\pi$ having an edge in common with the unbounded domain $X_{0}$ such that any separating curve for $\pi$ lies entirely in $X_{0}$ and $X_{1}$.

In a standard projection, condition (2) in the definition of elementary is violated only by the domains $X_{0}$ and $X_{1}$.

Given any projection $\pi$ of a knot $K$ we proceed as follows to obtain a standard projection of $K$. Note that $\pi$ cannot have any vertex lying on fewer than three distinct domains since $K$ is a knot. Now, if $\pi$ contains a vertex $v$ such that only three distinct domains meet at $v$, then a simple closed curve $\Delta$ may be drawn intersecting $\pi$ only in $v$ and separating $\pi$ into two parts. By appropriately turning over the part of the projection inside $\Delta$, the crossing $v$ can be eliminated. (See Figure 1.) (This untwisting is operation $\Omega .4$ of Reidemeister [3] and is discussed fully there.) If $\pi$ is alternating then so is the new projection. Since this operation is effective and reduces the number of crossings, in a finite number of steps we reach a projection having four distinct domains meeting at each vertex. 


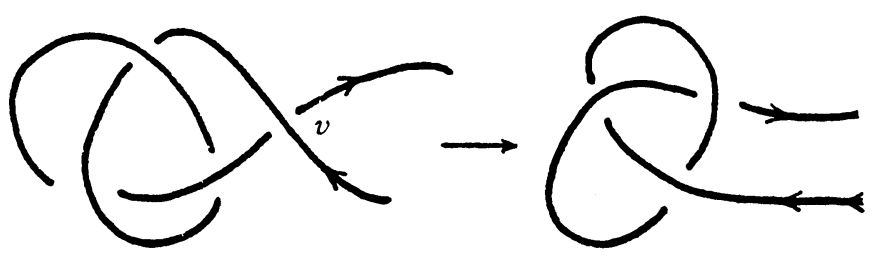

FiguRE 1

Now suppose that (ii) of the definition of standard fails. Choose any bounded domain $X_{1}$ which has an edge $e$ in common with the unbounded domain $X_{0}$. If $\Gamma$ is a separating curve lying in any pair of domains $X_{a}$ and $X_{b}$ other than $X_{0}$ and $X_{1}$, then $\Gamma$ is called a bad separating curve. If $\Gamma$ is a bad separating curve, then any vertex $v$ on an edge of $\pi$ intersected by $\Gamma$ is called a bad vertex. Clearly $v$ lies on $X_{a}$ and $X_{b}$.

Let $\Gamma$ be a bad separating curve. It may be that $\Gamma$ contains $X_{1}$ in its interior. Then, since $X_{1}$ is adjacent to $X_{0}, \Gamma$ must lie in $X_{0}$ and some $X_{a}$. Hence there is another bad separating curve $\Gamma^{\prime}$ lying in $X_{0}$ and $X_{a}$ which contains the part of $\pi$ exterior to $\Gamma$. If $\Gamma$ does contain $X_{1}$ in its interior we replace $\Gamma$ by $\Gamma^{\prime}$ and change notation. We thus may assume that $\Gamma$ is a bad separating curve which does not contain $X_{1}$ in its interior.

The following is an intuitive description of what is essentially Schubert's argument. Take a sphere $S$ enclosing that part of the knot whose projection is enclosed by $\Gamma$. The knot pierces $S$ exactly twice. Shrink $S$ and its interior to a "suitably small" size. Regard the point at which the knot enters $S$ as being fixed. By stretching the knot, pull $S$ along the knot until reaching an "unused" portion of the original edge $e$. (See Figure 2.)

In view of the previous discussion we define a transfer operation on the projection $\pi$ as follows. Let $\Gamma$ be a bad separating curve not containing $X_{1}$ in its interior. Let $\pi^{\prime}$ be the part of $\pi$ interior to $\Gamma$ and let $\pi^{\prime \prime}$ be the part of $\pi$ exterior to $\Gamma$. Obtain $\pi^{*}$ from $\pi$ as follows.

(1) Delete $\pi^{\prime}$ and replace it by an arc interior to $\Gamma$ joining the ends of $\pi^{\prime \prime}$.

(2) Delete a small arc $\delta$ from the original edge $e$. Replace $\delta$ by a curve $\Sigma$ (geometrically) similar to $\pi^{\prime}$ but small enough so that $\Sigma$ does not intersect $\pi$ except at the joining points and such that orientation in going around the knot is preserved.

(3) Since the projection enters $\Gamma$ once and leaves $\Gamma$ once, each vertex interior to $\Gamma$ is traversed twice, once as an undercrossing and once as an overcrossing. Hence, if $\pi$ is alternating then $\pi^{\prime}$ is alternating and similarly $\pi^{\prime \prime}$ is alternating. When $\Sigma$ is moved to the edge $e$, there may be a pair of consecutive undercrossings (or overcrossings) consisting of the last vertex traversed in $\Sigma$ and the next vertex traversed. If this happens, turn 

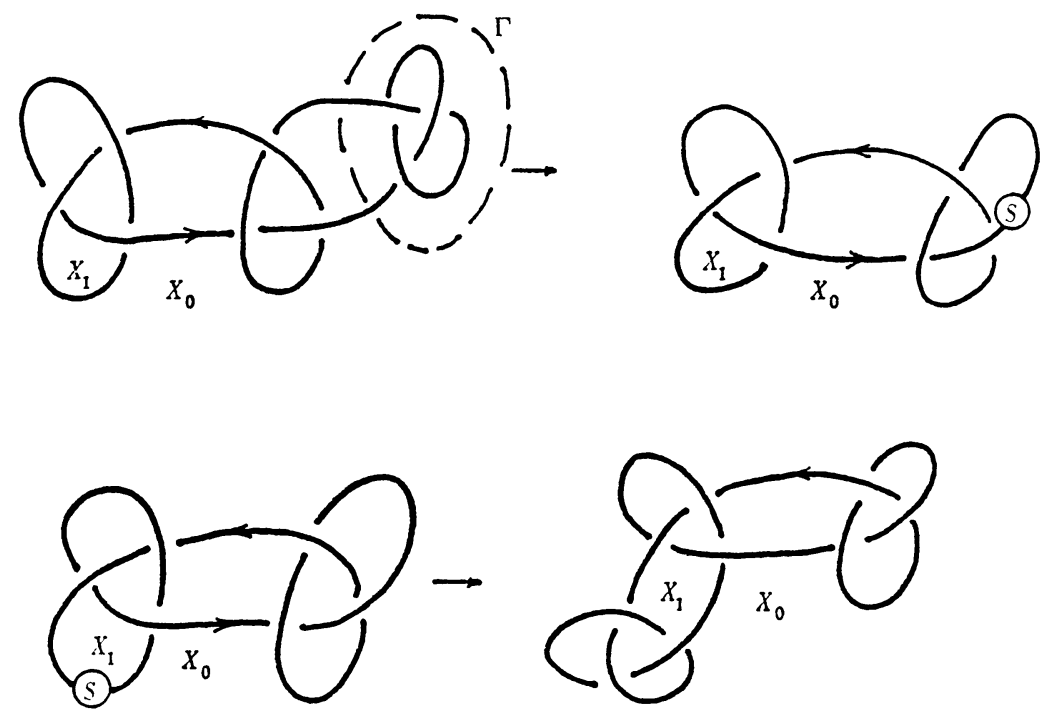

FiguRE 2

over $\Sigma$. This reverses overcrossings and undercrossings in $\Sigma$ and the projection $\pi^{*}$ is now alternating.

Now a transfer operation does not change the number of vertices but does reduce the number of bad vertices. Hence, after a finite number of transfers we obtain a standard projection. The fact that the knot type is not changed is justified by the intuitive description preceding the definition in which we perform allowable deformations on the knot.

DEFINITION. Let $\pi$ be a projection which is standard but not elementary. Remove a single (interior) point from each edge $e_{i}$ common to the boundaries of $X_{0}$ and $X_{1}$. This divides $\pi$ into parts $J_{i}$ which, if their loose ends are joined, are elementary projections. We call the $J_{i}$ the elementary parts of $\pi$.

Let us recall what Weinbaum proves. He uses the Dehn presentation of a knot group. (We will use the same letters to denote both the domains of a projection and the corresponding generators of the group.) Weinbaum shows that, for any projection of a knot, if the relator $X_{0}$ is deleted the resulting group, $G$, is the free product of the knot group and an infinite cycle. We will work with the same group $G$ and speak of the Dehn presentation of $G$. Weinbaum used the hypothesis of primeness only to ensure that the projection is elementary. He proved that if the projection is elementary and alternating then the Dehn presentation of $G$ satisfies the small cancellation conditions $\mathrm{C}(4)$ and $\mathrm{T}(4)$. (Weinbaum denotes the 
triangle condition by $T_{3}$ while we use $T(4)$.) The hypothesis that the projection is elementary and alternating is used only to verify $C(4)$. Although not stated explicitly in Weinbaum, the proof supplied shows that for any projection of a knot, the Dehn presentation of $G$ satisfies $T(4)$.

Let $R$ be a symmetrized set of relators. A sequence $u_{1} r_{1} u_{1}^{-1}, \cdots, u_{n} r_{n} u_{n}^{-1}$ of conjugates of elements of $R$ is called minimal if the product $w=$ $u_{1} r_{1} u_{1}^{-1} \cdots u_{n} r_{n} u_{n}^{-1}$ is not a product of fewer conjugates of elements of $R$. In constructing diagrams to study word and conjugacy problems one need only consider diagrams of minimal sequences. (See Schupp [5].) In the present context we consider finite symmetrized sets $R$ of defining relators each of which has length four. We say that $R$ satisfies $\mathrm{C}(4)$ and $\mathrm{T}(4)$ for minimal sequences if

(1) If two elements $r_{1}, r_{2}$ of $R$ cancel two or more letters then either $r_{1} r_{2}=1$ or $r_{1} r_{2}$ is already an element of $R(\mathrm{C}(4))$.

(2) If $r_{1}, r_{2}, r_{3}$ are elements of $R$ with cancellation in all the products $r_{1} r_{2}, r_{2} r_{3}$ and $r_{3} r_{1}$, then $r_{1} r_{2} r_{3}$ is a product of two or fewer elements of $R$ (T(4)).

It is easy to see that if $R$ satisfies $\mathrm{C}(4)$ and $\mathrm{T}(4)$ for minimal sequences then a diagram of a minimal sequence is a $(4,4)$ map. Thus such a group has solvable word and conjugacy problems exactiy as if the relators satisfied $\mathrm{C}(4)$ and $\mathrm{T}(4)$ in the absolute sense. We will show that if $K$ is an alternating knot then the group defined by Weinbaum can be presented so that $\mathrm{C}(4)$ and $\mathrm{T}(4)$ are satisfied for minimal sequences.

Let $K$ be any alternating knot and let $\pi_{1}$ be any alternating projection of $K$. If $\pi_{1}$ is not standard apply the procedure of the first part of the paper to obtain a standard alternating projection $\pi$ of $K$. We assume that $\pi$ is standard but not elementary (otherwise Weinbaum's argument is immediately applicable).

In each elementary part $J_{i}$ there are two crossings on $X_{0}$ and $X_{1}$. Since $\pi$ is alternating, without loss of generality we may assume that the one of these which is the first vertex traversed in the parametrization of $\pi$ is traversed by an overcrossing curve. This vertex is called the overcrossing of $J_{i}$. Similarly the other is called the undercrossing of $J_{i}$. We define domains $U_{i}, V_{i}, P_{i}, Q_{i}$ as follows: $U_{i}$ and $V_{i}$ are the domains other than $X_{0}$ and $X_{1}$ on the undercrossing of $J_{i}$ with $U_{i}$ adjacent to $X_{0}$ and $V_{i}$ adjacent to $X_{1} . P_{i}$ and $Q_{i}$ are the domains other than $X_{0}$ and $X_{1}$ on the overcrossing of $J_{i}$ with $P_{i}$ adjacent to $X_{0}$ and $Q_{i}$ adjacent to $X_{1}$. (See Figure 3.) It is possible that $P_{i}=U_{i}$ or $Q_{i}=V_{i}$ but if both equations held then either $P_{i}$ and $Q_{i}$ are adjacent along two edges, contradicting $J_{i}$ being elementary, or $K$ consists of two linked curves; hence not both can hold.

We have the following results from Weinbaum. 


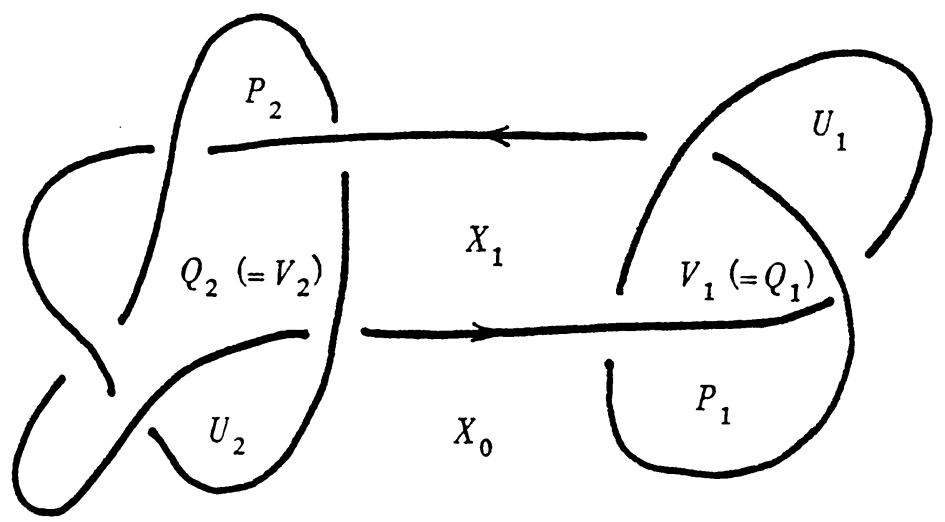

FIGURE 3

(1) The relators arising from each elementary part satisfy C(4) among themselves.

(2) The relators as a whole satisfy $\mathrm{T}(4)$.

We note that independent of the orientation of the knot, in the Dehn presentation for $G$, the symmetrized set determined by the relator corresponding to the overcrossing on $J_{i}$ is determined by $X_{1} X_{0}^{-1} P_{i} Q_{i}^{-1}$, while that corresponding to the undercrossing is determined by $X_{0}^{-1} X_{1} V_{i}^{-1} U_{i}$. The presentation we consider is the Dehn presentation augmented by the symmetrized set determined by relators $P_{i} Q_{i}^{-1} Q_{j} P_{j}^{-1}$ (which we call an overcrossing relator) and $V_{i}^{-1} U_{i}\left(i_{j}^{-1} V_{j}\right.$ (called an undercrossing relator) with $i, j$ ranging over all pairs of distinct indices of elementary parts of $K$. We call these additional relators the derived relators.

Two observations seem to give some idea of why this proof succeeds while extension of this method to nonalterating knots may be impossible. First we note that if two relators from distinct elementary parts have any generators in common these must be among $X_{0}$ and $X_{1}$. Second, for any derived overcrossing relator, in reading two successive occurrences of generators from the same elementary part, the exponents are positive followed by negative. In reading successive occurrences of generators from different elementary parts the exponents are negative then positive. For the derived undercrossing relators the exponent patterns are opposite. To verify $\mathrm{C}(4)$ we must show that if $r_{1}$ and $r_{2}$ are relators such that $r_{1} r_{2}$ has length four or less then $r_{1} r_{2}$ is either trivial or another relator. From Weinbaum's work we may assume that $r_{1}$ is a derived relator. The argument is symmetric for over and undercrossings so we will assume that $r_{1}$ is in the symmetrized set determined by $P_{i} Q_{i}^{-1} Q_{j} P_{j}^{-1}$.

Case 1. $r_{1}$ is $P_{i} Q_{i}^{-1} Q_{j} P_{j}^{-1}$. Now if $r_{2}$ is a relator corresponding to a crossing on $J_{j}$ then it must be $P_{j} Q_{j}^{-1} X_{1} X_{0}^{-1}$ by $\mathrm{C}(4)$ on relators from 
elementary parts, and then $r_{1} r_{2}=P_{i} Q_{i}^{-1} X_{1} X_{0}^{-1}$. If $r_{2}$ is a derived overcrossing relator it must be $P_{j} Q_{j}^{-1} Q_{k} P_{k}^{-1}$ and $r_{1} r_{2}$ is $P_{i} Q_{i}^{-1} Q_{k} P_{k}^{-1}$. Now suppose $r_{2}$ is determined by a derived undercrossing relator. Then it must begin $U_{j} V_{j}^{-1}$ where $U_{j}=P_{j}, V_{j}=Q_{j}^{-1}$, but this exponent pattern is impossible for an undercrossing relator.

Case 2. $r_{1}$ is $Q_{i}^{-1} Q_{j} P_{j}^{-1} P_{i}$. Here clearly $r_{2}$ must be a derived relator. If it is determined by an overcrossing relator it must be $r_{1}^{-1}$. If it is determined by an undercrossing relator it must begin $U_{i}^{-1} U_{j}$ where $U_{i}=P_{i}$, $U_{j}=P_{j}$. But again, no such relator exists because of the exponent pattern.

To verify $\mathrm{T}(4)$ we may assume that $r_{1}$ is a derived relator and that $r_{1}, r_{2}, r_{3}$ are such that each of $r_{1} r_{2}, r_{2} r_{3}$ and $r_{3} r_{1}$ have precisely one cancelling letter.

Case 1. $r_{1}$ is $P_{i} Q_{i}^{-1} Q_{j} P_{j}^{-1}$. If $r_{2}$ is an overcrossing relator then the product $r_{1} r_{2}$ is not minimal. Thus this case may be ignored.

Subcase (i). $P_{j}=U_{j}$ and $r_{2}=U_{j} U_{k}^{-1} V_{k} V_{j}^{-1}$. Then $r_{3}$ has the form $V_{j} A B P_{i}^{-1}$. Since $P_{j}=U_{j}$, we must have $V_{j} \neq Q_{j}$. Hence, $r_{3}$ is the derived undercrossing relator $V_{j} V_{i}^{-1} U_{i} U_{j}^{-1}$. But then $P_{i}=U_{j}$, contradicting the uniqueness of the elementary part in which $P_{i}$ lies.

Subcase (ii). The situation where $r_{3}$ is a derived relator is the same as that where $r_{1}$ and $r_{2}$ are derived relators. Thus, we may now suppose that neither $r_{\varepsilon}$ nor $r_{3}$ are derived relators. Then $r_{2}$ comes from the elementary part $J_{j}$ and $r_{3}$ comes from $J_{i}$. Thus the generator cancelled in the product $r_{2} r_{3}$ is either $X_{0}$ or $X_{1}$. Now $r_{2}$ must begin with $P_{j}$. The knot projection is a graph all of whose vertices have degree four. By a standard chromatic lemrna of graph theory, its domains may be "properly" twocolored. Thus if $D$ and $E$ are opposite domains at one vertex they cannot be adjacent at another vertex. Since $P_{j}$ is adjacent to $X_{0}$ at one vertex the last letter in $r_{2}$ must be $X_{0}^{-1}$. By $\mathrm{C}(4)$ in the elementary part $J_{j}, r_{2}=$ $P_{j} Q_{j}^{-1} X_{1} X_{0}^{-1}$. Hence, $r_{3}=X_{0} A B P_{i}^{-1}$. By C (4) in $J_{i}, r_{3}=X_{0} X_{1}^{-1} Q_{i} P_{i}^{-1}$. Thus $r_{2} r_{3}$ is not minimal.

Case 2. $r_{1}$ is $Q_{i}^{-1} Q_{j} P_{j}^{-1} P_{i}$. As before, if $r_{2}$ is a derived overcrossing relator then the product $r_{1} r_{2}$ is not minimal.

Subcase (i). If $r_{2}$ is a derived undercrossing relator then $P_{i}=U_{i}$ and $r_{2}=U_{i}^{-1} V_{i} V_{k}^{-1} U_{k}$. Then $r_{3}$ is $U_{k}^{-1} A B Q_{i}$. Since $Q_{i} \neq V_{i}, r_{3}=P_{i}^{-1} P_{j} Q_{j}^{-1} Q_{j}$, yielding the contradiction $P_{i}=U_{k}$ where $i \neq k$.

Subcase (ii). Suppose that neither $r_{2}$ nor $r_{3}$ is derived. Then both come from the elementary part $J_{i}$. If $r_{1}, r_{2}, r_{3}$ all cancel, then so do $r_{1}^{\prime}, r_{2}, r_{3}$ where $r_{1}^{\prime}=Q_{i}^{-1} X_{1}^{-1} X_{0}^{-1} P_{i}$. This violates $\mathrm{T}(4)$ unless one of $r_{2}$ or $r_{3}$ is $\left(r_{1}^{\prime}\right)^{-1}$. This assumption yields a contradiction since it implies that either $r_{2}$ or $r_{3}$ is not cyclically reduced. For example, if $r_{2}=\left(r_{1}^{\prime}\right)^{-1}$, then $r_{3}$ must begin with $Q_{i}^{-1}$ and end with $Q_{i}$ for cancellation to occur in $r_{2} r_{3}$ and $r_{3} r_{1}$. This completes the proof of the theorem. 


\section{REFERENCES}

1. K. I. Appel, The conjugacy problem for tame alternating knot groups is solvable, Notices Amer. Math. Soc. 18 (1971), 942. Abstract \#71T-A227.

2. R. C. Lyndon, On Dehn's algorithm, Math. Ann. 166 (1966), 208-226. MR 35 \#5499.

3. K. Reidemeister, Knotentheorie, Ergebnisse der Mathematik, Vol. 1, no. 1, Springer, Berlin, 1932.

4. H. Schubert, Der eindeutige Zerlegbarkeit eines Knotens in Primknoten, S.-B. Heidelberger Akad. Wiss. Math.-Nat. Kl. 1949, no. 3, 57-104. MR 11, 196.

5. P. E. Schupp, On Dehn's algorithm and the conjugacy problem, Math. Ann. 178 (1968), 119-130. MR 38 \#5901.

6. C. Weinbaum, The word and conjugacy problems for the knot group of any tame prime alternating knot, Proc. Amer. Math. Soc. 30 (1971), 22-26.

Department of Mathematics, Univeristy of Illinois, Urbana, Illinois 61801 